\title{
Grain boundaries (moderator's comment)
}

\author{
H.O.K. Kirchner \\ Institut für Festkörperphysik, Universität Wien, 1090 Boltzmanngasse 5, Vienna, Austria
}

\begin{abstract}
Résumé. - Les joints de grains sont des obstacles effectifs pour le mouvement des dislocations. Les contraintes d'incompatibilité associée causent du glissement multiple. Dans la vicinité du joint le cristal se trouve dans un état avancé du durcissement. Le cisaillement transgranulaire est quantitativement peu important.

Abstract. - Grain boundaries are an effective obstacle to dislocation motion. The resulting incompatibility stress causes multiple slip. Adjacent to the grain boundary the crystal is in an advanced stage of hardening. Transgranular slip is quantitatively unimportant.
\end{abstract}

1. Transmission of slip vs. hardening of the grainboundary zone

An area of research where contact between physicists and mechanics people is not only possible but even necessary is the question of the importance of transgranular (as opposed to intragranular) slip. By transgranular slip we mean that slip occurs in one grain, proceeds up to the grainboundary and continues into the other grain, possibly even on another slip system. Intragranular slip means that slip does not proceed across the grain-boundary. From the work presented by George [1] and Rey [2] one concludes that even in bicrystals which are favourably oriented for transmission of slip from one grain to the other, such transgranular slip is not very important as far as the overall transformation is concerned. The first to discuss this questions seems to have been Hirth [3]. He proposed a model in which a hardened zone exists next to the grain-boundary. The polycrystal is considered as a twophase material in which, grosso modo, the interior of the grains remain in a state of deformation comparable to a single crystal at the same strain, while the region adjacent to the boundaries carries a flow stress that would be characteristic of a much more advanced stage of deformation in a single crystal. The hardening there is due to the same processes that cause stage II or III in a single crystal, but here in the polycrystal dislocations do not block each other, as in a single crystal, but are blocked by the grain-boundary and only subsequently block each other. As a consequence of this even a small amount of deformation cannot be provided by the operation of one slip-system, but the incompatibility at the boundary necessitates the operation of several slip systems in each grain, with all the hardening entailed by it. Martinez-Hernandez, Kirchner, Korner, George and Michel [4] have, as a matter of fact, given evidence for cross-slip, multiple cross-slip, secondary and tertiary slip near the interface of a silicon bicrystal. Korner, Martinez-Hernandez, George and Kirchner [5] found also noncompact glide under the same circumstances. With some experimental evidence already present, it would be interesting to apply Mughrabi's [6] two phase model of hardening to this situation. The film of El'Mrabat et al [7] shows the hardening behaviour of the grain boundary zone in situ in iron in a dynamic situation. Although one might suspect that in the bcc structure a wider variety of slip systems should be available from the outset, and transgranular slip should be easier than, for example, in silicon, it was never observed.

2. Crystallography of transmission of slip

Even if transmission of slip from one grain into the other is quantitatively unimportant, the details of the process are of great interest from a crystallographic point of view. As described by George [1] the bulk dislocation (or rather its leading partial) first has to enter the grain-boundary. After that it can react with structural units or defects of the boundary. Finally it might leave the boundary by a similar reaction in which a lattice dislocation is formed in the other grain.

El Kajbaji [8] and El Kajbaji, Thibault-Desseaux, Martinez-Hernandez, Jacques and George [9] give an example of such a decomposition of a bulk Burgers vector into two basic vectors of the DSC lattice. In their case the boundaryis perfect and free of extrinsic dislocations.

If the boundary is not perfect and contains it- 
self some dislocations,passing of a bulk dislocation across the boundary will involve some reaction with the extrinsic dislocation already present. Figs. 1 and 2 , kindly provided by $W$. Skrotzki [10] show such a reaction in germanium. After entering the boundary the matrix dislocation decomposes. The decomposition products in turn react with dislocations already present in the boundary, and finally the new reaction product exits into the other grain.

3. Decomposed and dispersed dislocations

Fractional vacancies

Pumphrey and Gleiter $[13,14]$ were the first to observe that dislocations arriving from the bulk and entering the grain-boundary can disperse either by glide [15] or climb $[16,17]$. The first process, glide, is similar to a continous decomposition of a discrete Burgers vector as envisaged in the Peierls model for the bulk.

The decomposition into a continous distribution of Burgers vectors has never been seen so far, but since the high resolution work was done on silicon and germanium where continuous spreading is neither expected nor observed even for bulk dislocation.

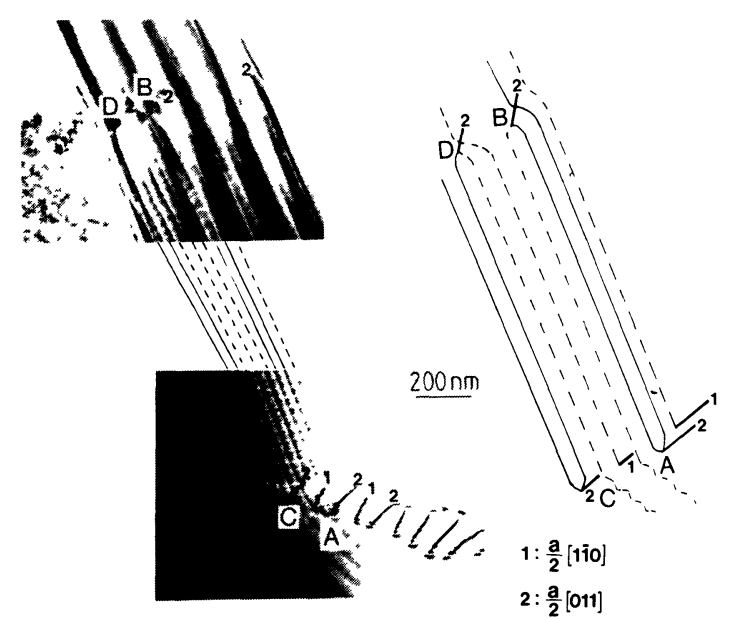

Figure 1. Passage of matrix dislocation with $\mathrm{b}=\mathrm{a}$ [011]/2 through a $\Sigma=51$ grain boundary in germanium. Micrograph and schematic representation. The screw dislocations (2) decompose into two secondary dislocations each at $A$ and $C$. After recombining at $B$ and $D$ they exit into the second grain. The recombination is actually the result of an interaction between one of the dissociation products and another dislocation of a set which was already present in the interface. See also refs. [11] and [12].

this is not surprising. Presumably the continuous spreading of grain boundary dislocations will be observed in metals where also the bulk cores are dispersed. The second process, climb, is distinctly different from what can occur in the bulk, and, as a matter of fact, is impossible in the bulk. From the experimental observation that the contrast of extrinsic dislocations disappears within a certain time in the electron microscope, one concludes that the dislocation must either have become delocalized over a region of the order of the extinction distance or must have decomposed discretely into invisibly small Burgers vectors.

If the Burgers vector of the dislocation is perpendicular to the interface, this can occur only by conservative or nonconservative climb. The fact that the discrete Burgers vector decomposes into a discontinuous (or even continuous) distribution of Burgers vectors which are fractions of the bulk one implies that there must have been absorption of fractional point defects within the boundary. Absorption of one or more complete bulk vacancies is necessary for the climb of one DSC lattice dislocation by one period of the coincidence lattice. Climb occurs, however, by steps smaller than that. The fractional vacancy mentioned is just a vacancy of the DSC lattice, which is denser than the bulk one, but not fully occupied. For example, in a $\Sigma=9$ boundary the DSC vacancy is one ninth of the bulk vacancy [18].

\section{Crystallography vs. elasticity}

By now crystallographic studies of grainboundary structures abound in the literature. Molecular statics calculations [19] show that,

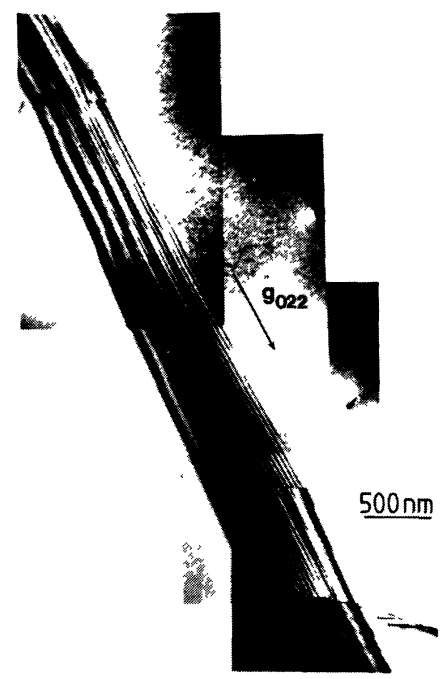

Figure 2. Overall view of Fig. 1. Entrance and exit of a bulk dislocation into or from a boundary might be a few thousand nanometers apart. Kindly provided by W. Skrotzki[10].

just as a bulk crystal has a particular structure, each grain-boundary has a particular structure, with unit motifs, periodicities and all the other ingredients of crystallography. The subject of interfacial crystallography has been considerably advanced by Bollmann $[20,21]$ and others $[22,23]$. One might ask if the crystallography of an interface is really important for its mechanical behaviour. The answer probably has to be a qualified yes, insofar as impurities and point defects are important for the deformation process. Obviously, when at low temperatures transgranular slip is considered as unimportant anyway, the particular structure of the interface which is not transversed is of little importance. If, however, at high temperatures impurities or point defects are involved in the deformation process, their interaction with and within the grain boundary might be all important. 
Deformation within the grains, on the other hand, might well beinfluenced by the fact that dislocations find themselves in the proximity of an interface which modifies their elastic fields. Interaction of the dislocations among each other is modified as well. The state of the art of interfacial elasticity is far from satisfactory, suffice it to mention a few features which are qualitatively (and not only quantitatively) different from the bulk situation: Firstly, incompatibility stresses caused by an interface cannot be defined as neatly by projection methods as internal or external stresses (Simmons and Bullough [24]. Secondly, unlike as in the bulk, where only dislocations, and forces can carry configurational forces (Cherepanov [25], Kirchner [26]), the interface presents an elastic inhomogeneity and thus can carry a force as well (Eshelby [27]). A consequence of this is that an interfacial dislocation is always associated with a force enacted by one grain unto the other. The formal solution available for a dislocation along the interface between two semiinfinite crystals (Barnett and Lothe [28], Kirchner and Lothe [29]) clearly shows this effect.

A mathematical consequence of this phenomenon is that the integral equation for an interfacial crack becomes a Fredholm equation of the second kind (rather than of the first kind, as for the bulk). Such equations are equivalent to Hilbert problems and have oscillatory solutions (Willis
[30], Rice and Sih [31]). This leaves some hitherto unresolved questions about the proper definition of a stress intensity vector for interfacial cracks.

\section{Special vs. nonspecial boundaries}

The group at St. Etienne was the first to study extensively the behaviour of bicrystals and the interface between them. Their technique allowed the production of crystallographically well defined interfaces. The Cornell group extended this technique to the production of thin bicrystals suitable for electron microscopy. This led to extensive work on defects in or near these boundaries which are special because of their mode of production.

The question arises how relevant results obtained on special boundaries are for the boundaries present in a polycrystal. In spite of the fact that it is far from clear that all or a sizeable proportion of all boundaries in a polycrystal are random, it would be extremely interesting to know if, as far as the deformation behaviour of polycrystals is concerned, the behaviour of special and nonspecial boundaries is different. The answer is probably no if no transgranular slip occurs. On the other hand, as far as chemical effects are concerned, the crystallographic structure (special or not) is of great importance.
[1] George, A., this volume.

[2] Rey, C., This volume.

[3] Hirth, J.P., Met. Trans. 3 (1972) 3047.

[4] Martinez-Hernandez, M., Kirchner, H.O.K., Korner, A., George, A., and Michel, J.P., Philosoph. Mag. A (1987) in print.

[5] Korner, A., Martinez-Hernandez, M., George, A., and Kirchner, H.O.K., Philosoph. Mag. Letters 55 (1987) 105.

[6] Mughrabi, H., Mat. Sci. Engineering 85 (1987) 15 .

[7] El'Mrabat, B., Priester, L., Valle, R., Jouniaux, A., Marraud, A., this volume.

[8] El Kajbaji, M., Thesis (1986) Université de Grenoble.

[9] E1 Kajbaji, M., Thibault-Desseaux, J., Martinez-Hernandez, M. , Jacques, A., and George, A., Rev. Phys. Appl. (1987) to be published.

[10] Skrotzki, W., private communication.

[11] Skrotzki, w., Wendt, H., Carter, C.B., and Kohlstedt, D.L., this volume.

[12] Skrotzki, W., Wendt, H., Carter, C.B., and Kohlstedt, D.L., Proc. Mat. Res. Soc. Symp. 41 (1985) 261 .

[13] Pumphrey, P.H., and Gleiter, H., Philosoph. Mag. 30 (1974) 593.
[14] Pumphrey, P.H., and Gleiter, H., Philosph. Mag. 32 (1975) 881 .

[15] Lojkowski, W., Kirchner, H.O.K., and Grabski, M., Scripta Met. 11 (1977) 1127.

[16] Valiev, R.z., Gertsman, V. Yu., Kaibyshev, O.A., and Khannanov, Sh.Kh., phys. stat. sol. (a) $\underline{77}$ (1983) 97

[17] Valiev, R.z., Gertsman, V.Yu., and Kaibyshev, O.A., phys. stat. sol., (a) 77 (1983) 177.

[18] Thibault-Desseaux, J., private communication.

[19] Vitek, V., Journal de Physique C6-43 (1982) 150.

[20] Bollmann, w., Crystal Defects and Crystalline Interfaces, Springer, Berlin (1970).

[21] Bollmann, W., Crystal lattices, Interfaces, Matrices, W. Bollmann, Genève (1982).

[22] Portier, R., Thalal, A., and Gratias, D., in: Les joints de grains dans les matériaux, les éditions de physique (1985) 69.

[23] Grimmer, H., and Warrington, D.H., Acta cryst. A 43 (1987) 232 .

[24] Simmons, J.A. and Bullough, R., in: Proc. Conf.Fundamental Aspects of Dislocation Theory, Gaithersburg, 1969, eds. J.A. Simmons, R. de Wit and R. Bullough (Nat. Bur. Stand. Spec. Publ. 317, vol. I, Washington) p. 89.

[25] Cherepanov, G.P., Engin. Fract. Mech. 14 (1981) 39 . 
[26] Kirchner, H.O.K., in: Materials Science Monographs 20. The Structure and Properties of Crystal Defects. Edited by V. Paidar and L. Lejcek, Elsevier, Amsterdam (1984), p. 370.

[27] Eshelby, J.D., Phil. Trans. A 244 (1951) 877.

[28] Barnett, D.M., and Lothe, J., J. Phys. F: Metal Phys. 4 (1974) 1618.

[29] Kirchner, H.O.K., and Lothe, J., Philosph. Mag. A (1987) in print.
[30] Willis, J.R., J. Mech. Phys. Solids 19 (1971) 353.

[31] Rice, J.R., and Sih, G.C., J.Appl.Mech. 32 (1965) 418.

Acknowledgement - This work was supported by the Hochschuljubiläumsstiftung der stadt Wien. 